\title{
TUNING-AMÉRICA LATINA: \\ UN PROYECTO DE LAS UNIVERSIDADES
}

\author{
Julia González $(*)$, Robert Wagenaar $(* *)$, \\ Pablo Beneitone $(* * *)$
}

SÍNTESIS: El proyecto Tuning-América Latina es una iniciativa de las universidades para las universidades. Se busca iniciar un diálogo para intercambiar información y para mejorar la colaboración entre las instituciones de educación superior, favoreciendo el desarrollo de la calidad, de la efectividad y de la transparencia. Con el trabajo de las 62 instituciones de educación superior de los 18 países latinoamericanos participantes se espera que, en los dos próximos años, se identifiquen puntos de referencia común en diferentes áreas del conocimiento. Estos puntos identificados son necesarios para tender los puentes destinados al reconocimiento de las titulaciones en la región y con otras regiones del planeta.

SÍNTESE: 0 projeto Tuning-América Latina é uma iniciativa das universidades para as universidades. Procura-se iniciar um diálogo para trocar informação e para melhorar a colaboração entre as instituições de educação superior, favorecendo o desenvolvimento da qualidade, da efetividade e da transparência. Com o trabalho das 62 instituições de educação superior dos 18 países latino-americanos participantes, esperase que, nos dois próximos anos, se identifiquem pontos de referência comum em diferentes áreas do conhecimento. Estes pontos identificados são necessários para alargar os canais destinados ao reconhecimento das titulações na região e com outras regiões do planeta.

(*) Co-coordinadora del Proyecto Tuning, Universidad de Deusto, Bilbao, España.

(**) Co-coordinador del Proyecto Tuning, Universidad de Groningen, Países

Bajos. Bilbao, España.

(***) Gestor del Proyecto Tuning-América Latina, Universidad de Deusto, 


\section{INTRODUCCIÓN}

El proyecto Tuning-América Latina 2004-2006 surge en un contexto de intensa reflexión sobre educación superior, tanto a nivel regional como internacional. Hasta ese momento Tuning había sido una experiencia exclusiva de Europa, un logro de más de 135 universidades europeas, que, desde el año $2001^{2}$, llevan adelante un intenso trabajo dirigido a la creación del Espacio Europeo de Educación Superior.

Tuning implicó en Europa un gran reto para las instituciones de educación superior, ya que permitió la creación de un entorno de trabajo para que los académicos pudieran llegar a puntos de referencia, de comprensión y de confluencia. Se generó un espacio que permitió «acordar», «templar», «afinar» las estructuras educativas en cuanto a las titulaciones, de manera que pudieran ser comprendidas, comparadas y reconocidas en el área común europea. Tuning quiere reflejar esa idea de búsqueda de puntos de acuerdo, de convergencia y de entendimiento mutuo, para facilitar la comprensión de las estructuras educativas. Estos puntos de referencia identificados son precisos para tender los puentes que sirvan para el reconocimiento de las titulaciones.

Hoy Tuning es una metodología internacionalmente reconoci$\mathrm{da}^{3}$, una herramienta construida por las universidades para las universidades, un instrumento que nos permite pensar que el Espacio de Educación Superior Europeo puede ser una realidad cada vez más cercana. Europa está transitando su camino de integración, y Tuning ha facilitado parte de ese proceso.

\section{2. ¿POR QUÉ TUNING-AMÉRICA LATINA?}

Dado este contexto, tal vez la primera inquietud que surge sea: ¿por qué un proyecto Tuning-América Latina? La respuesta puede ser results_es.htm.

1 Ver: http://europa.eu.int/comm/europeaid/projects/alfa/information/

2 Tuning está culminando su segunda fase en Europa (2003-2004), y ya ha sido aprobada por la Comisión Europea una tercera fase (2005-2006), que comenzará en enero de 2005.

${ }^{3}$ En el documento preparatorio del Comunicado de Berlín se menciona de manera destacada el proyecto Tuning como una de las actividades más importantes que se están llevando adelante en relación con el proceso de Bolonia. 
múltiple y variada, aunque no deberíamos dejarnos llevar por elucubraciones complejas; lo mejor es observar el núcleo de la problemática que enfrentan las universidades europeas en este momento, y contrastarlo con las que tienen por delante las latinoamericanas. En primer lugar, la necesidad de compatibilidad, de comparabilidad y de competitividad de la educación superior no es una aspiración exclusiva de Europa. EI actual proceso de gl obalización al que asistimos está signado, entre otras cosas, por la creciente movilidad de los estudiantes, que requiere información fiable y objetiva sobre la oferta de programas educativos. Además, hay que tener en cuenta la movilidad de los profesionales. Los empleadores actuales y futuros dentro y fuera de América Latina, exigirán conocer fehacientemente lo que significa en la práctica una capacitación o una titulación determinadas. Finalmente, en una etapa de internacionalización como la que estamos viviendo, la universidad, como actor social, se enfrenta a desafíos y a responsabilidades, sin tomar en cuenta de qué lado del Atlántico nos encontremos. Las instituciones de educación superior deben asumir un rol más protagónico en los distintos procesos que vamos construyendo como sociedad, y ese rol se vuelve crucial cuando hablamos de las reformas en educación superior.

La creación, la elaboración, la transmisión y la difusión del conocimiento, así como las demás funciones tradicionales de las universidades de investigar, de enseñar y de difundir nuevos conocimientos, les ha otorgado un papel central en esta sociedad del conocimiento. Así mismo, se les reconoce cada vez más su carácter de foco de desarrollo social y económico en muchos ámbitos, sobre todo en la formación de personal cualificado. Para ocupar un lugar en la sociedad del conocimiento la formación de recursos humanos es de fundamental importancia, y el ajuste de las carreras a las necesidades de las sociedades a nivel local y global es un elemento de relevancia innegable. Por eso, el esfuerzo sistematizado de pensar y de repensar juntos el horizonte académico (reconocimiento por la comunidad académica) y profesional (reconocimiento por los colegios y por los grupos profesionales), es una de las líneas principales del proyecto Tuning. Mantener a las universidades en diálogo constante con la sociedad, que es una parte siempre abierta al futuro, es relevante para cualquier realidad, aunque la sensibilidad y la adaptación a los diversos contextos sea por supuesto un requisito básico yal go que está presente en todo momento en el proyecto Tuning-América Latina.

La cooperación es otro de los aspectos que responde a la pregunta inicial. Los dos lados del Atlántico, como todas las regiones del 
planeta, están llamadas a colaborar. Tal necesidad no es nueva, pero sí más necesaria y más posible. Nuestro concepto de cooperación nada tiene que ver con la imposición ni con el colonialismo cultural. Tampoco estamos a favor de aquel que pueda vender «humo» de proyectos sin consistencia propia, que induzcan al engaño porque copian sin reconocer las fuentes, que pretendan existir sin tener el mínimo de entidad propia o de financiación, y que hagan invertir ilusión, tiempo y recursos en algo de dudosa realización.

Tuning sitúa su papel en una colaboración, que quisiéramos fuera honesta, en los siguientes términos:

- La oferta de esa colaboración es por descontado sólida, puesto que ha sido trabajada por muchos compañeros de academia y de profesión. Se dispone de una labor ya realizada y valiosa, porque hay más de doscientos equipos detrás. Se trata de un trabajo honrado, que reconoce fuentes y que transcribe los resultados.

- Cada equipo, cada país, está llamado a esa cooperación desde la especificidad de su contexto. Mantener la variedad no es menos importante que llegar al consenso. La incorporación de los 18 países de América Latina en el Tuning se hace desde sus propios contextos, ya que dichos contextos los necesita como base natural de actuación.

- Es un proyecto abierto, y la reflexión-incorporación de nuevos países y de nuevos contextos puede modificar el proyecto inicial. Esto es lo que entendemos por cooperación: estar abierto al impacto y al aprendizaje mutuo.

- Tuning está abocado a impactar con fuerza en los sistemas de reconocimiento académico, dato de no poca relevancia en el contexto de la movilidad mundial. Pero uno puede preguntarse acerca de cuántos sistemas podemos tener a nivel global y sobre la necesidad de crear sistemas variados. Sería más conveniente canalizar esfuerzos para llegar a conseguir sólo uno, entendido y elaborado por la mayoría, suficientemente abierto para que haya espacio para todos, pero también con capacidad definida para que los elementos cruciales 
de las carreras pudieran conocerse y «reconocerse» en los distintos sistemas educativos.

Creemos que una clara visión de futuro puede llevarnos a decisiones sobre dónde ponemos las energías y los recursos, que son bastante escasos para ser malgastados. En otras palabras, TuningAmérica Latina consiste en un trabajo conjunto que busca lenguajes y sistemas de reconocimiento de carácter transnacional y transregional.

De este modo, es importante plantear la idea de llevar adelante una propuesta como la de Tuning en América Latina, que surge en Europa pero que ahora es formulada por los latinoamericanos. Durante la IV Reunión de Seguimiento del Espacio Común de Enseñanza Superior de la Unión Europea, América Latina y el Caribe (UEALC) en Córdoba (España) en octubre de 2002, los representantes de América Latina que participaron en el encuentro, tras escuchar la presentación de los resultados de la primera fase del Tuning, expusieron la inquietud de proponer un proyecto similar con América Latina. Desde ese momento se empezó a preparar la iniciativa, que fue presentada por un grupo de universidades europeas y latinoamericanas ${ }^{4}$ a la Comisión Europea a finales de octubre de 2003. Podemos decir que la propuesta Tuning para América Latina es una idea intercontinental, un proyecto que se ha nutrido de los aportes de académicos tanto europeos como latinoamericanos. La idea de búsqueda de consensos es la misma, es única y universal; Io que cambia son los actores y la impronta que brinda cada realidad. Durante los meses de julio y agosto de 2004 (se concluirá en octubre) los 18 países latinoamericanos fueron visitados por los coordinadores de Tuning para explicar, dialogar y reajustar la propuesta con los ministerios de educación y/o las conferencias de rectores de esos países. Las sugerencias recibidas fueron ya incorporadas a la trayectoria inmediata o a un corto plazo en el proceso Tuning.

\footnotetext{
${ }^{4}$ Las ocho universidades latinoamericanas son: Universidad Nacional de La Plata (Argentina), Universidad Estadual de Campinas (Brasil), Universidad de Chile, Universidad de Costa Rica, Pontificia Universidad J averiana (Colombia), Universidad Católica Andrés Bello (Venezuela), Universidad Rafael Landívar (Guatemala) y Universidad de Guanajuato (México). Las siete universidades europeas son: Universidad de Deusto (España), Universidad de Groningen (Países Bajos), Universidad de Coimbra (Portugal), Universidad de Bristol (Reino Unido), Universidad de Pisa (Italia), Universidad de París IXDauphine (Francia) y Technische Universität Braunschweig (Alemania).
} 
Finalmente, y para completar las diferentes explicaciones que hacen posible pensar en un Tuning-América Latina, es importante señalar el interés de las universidades europeas por conocer cuáles serán las conclusiones a las que llegarán sus pares latinoamericanos. Existe una gran expectación por saber el grado de correlación que guardan los resultados que se alcanzaron en Europa con los que se obtengan en América Latina. Dichos resultados nutrirán aún más el debate existente y permitirán discutir en el futuro un perfil internacional de las titulaciones, combinando elementos identificados tanto en Europa como en América Latina.

\section{3. ¿QUÉ IMPLICA TUNING-AMÉRICA LATINA?}

El proyecto busca iniciar un debate cuya meta es identificar e intercambiar información, y mejorar la colaboración entre las instituciones de educación superior para el desarrollo de la calidad, la efectividad y la transparencia. La protección de la rica diversidad de la educación superior latinoamericana es fundamental en el proyecto, y bajo ninguna circunstancia se busca restringir la autonomía universitaria. Este punto es un pilar básico del proyecto. Uno de sus objetivos claves es el de contribuir al desarrollo de titulaciones fácilmente comparables y comprensibles «desde dentro», desde los objetivos que la titulación se marque, desde los perfiles buscados para los egresados en forma articulada y en toda América Latina. En la búsqueda de perspectivas que puedan facilitar la movilidad de los poseedores de títulos universitarios y profesionales en América Latina, yquizás también en Europa, el proyecto trata de alcanzar un amplio consenso a escala regional sobre la forma de entender los títulos desde el punto de vista de las actividades que los poseedores de dichos títulos serían capaces de desempeñar. De esta forma, el punto de partida del proyecto estaría en la búsqueda de puntos de referencia comunes, centrándose en las competencias y en las destrezas (basadas siempre en el conocimiento).

La alternativa de usar esos puntos de referencia y no definiciones de materias muestra un claro posicionamiento, ya que si los profesionales se van a establecer y a buscar empleo en otros países de América Latina, su educación tiene que tener un cierto nivel de consenso con respecto a puntos de referencia acordados conjuntamente, y que han sido reconocidos dentro de cada una de las áreas de las disciplinas específi- 
cas. Además, el uso de puntos de referencia deja espacio para la diversidad, para la libertad y para la autonomía.

Siguiendo su propia metodología, Tuning-América Latina tiene cuatro grandes líneas: competencias (genéricas y específicas de las áreas temáticas); enfoques de enseñanza, aprendizaje y evaluación; créditos académicos; y calidad de los programas.

En lo concerniente a la primera línea, se trata de identificar competencias compartidas que pudieran generarse en cualquier titulación, y que son consideradas importantes por ciertos grupos sociales. Hay algunas competencias, como la capacidad de aprender, la de análisis y de síntesis, etc., que son comunes a todas o a casi todas las titulaciones. En una sociedad en transformación, en la que las demandas se están reformulando de manera constante, las destrezas o competencias genéricas se hacen muy importantes. Aquí, en la primera línea, se analizan, además de las competencias genéricas, aquellas otras que se relacionan con cada área temática y que son esenciales para cualquier titulación, porque están relacionadas de forma concreta con el conocimiento específico de un área temática. Se conocen también como destrezas y competencias relacionadas con las disciplinas académicas, y son las que confieren identidad y consistencia a cualquier programa.

Las competencias difieren de una disciplina a otra. Para elaborar programas más transparentes y comparables a nivel latinoamericano, es necesario conseguir resultados del aprendizaje y competencias para cada titulación. La definición de estas competencias es responsabilidad de los académicos, no sin antes consultarla con otras personas interesadas en el tema dentro de la sociedad. Al definir competencias y resultados del aprendizaje de la manera indicada, se alcanzan puntos de referencia consensuad os que sientan las bases para garantizar la calidad, y una evaluación interna nacional e internacional.

En la segunda línea se propone preparar una serie de materiales que permita visualizar cuáles serán los métodos de enseñanza, aprendizaje y evaluación más eficaces para el logro de los resultados del aprendizaje y de las competencias identificadas. Eso implica llevar a cabo una mezcla novedosa de enfoques de enseñanza y aprendizaje para estimular -o para permitir que se desarrollen- las competencias que se diseñen en el perfil, como la independencia de criterio, la habilidad para comunicarse 0 el trabajo en equipo. Los cambios de enfoques y de objetivos de enseñanza y aprendizaje implican también las modificacio- 
nes correspondientes en los métodos de evaluación y en los criterios para evaluar la realización. Estos deben considerar no sólo el conocimiento y los contenidos sino las habilidades y destrezas generales. Cada estudiante debe experimentar una variedad de enfoques y tener acceso a diferentes contextos de aprendizaje, cualquiera que sea su área de estudio. Por supuesto, la transparencia y la capacidad de comparabilidad de los métodos y de los criterios para evaluar la realización son esenciales si queremos incrementar la garantía de la calidad. Si la primera línea del proyecto busca la definición de las competencias genéricas y específicas, ésta se propone el modo más adecuado de aprenderlas, de enseñarlas y de evaluarlas.

En la tercera línea se inicia una reflexión sobre el impacto y sobre la relación de este sistema de competencias con el trabajo del estudiante, y la de su medida y su conexión con el tiempo resultante medido en créditos académicos.

Finalmente, la cuarta línea propuesta asume que la calidad es una parte integrante del diseño del currículo basado en competencias, lo que resulta fundamental para articularla con las dos líneas anteriores. Si un grupo de académicos desea elaborar un programa de estudios o redefinirlo, necesita un conjunto de elementos para brindar calidad a esos programas y a esas titulaciones. Es necesario pensar cómo incorporar la calidad a los programas de estudio, y cómo demostrar que dicha calidad ha sido alcanzada. La confianza mutua entre las instituciones de educación superior y el reconocimiento de las titulaciones que éstas expidan, debe tener como soporte básico una metodología común y contrastada de evaluación de la calidad. La movilidad yel reconocimiento de estudios no sólo requieren un clima de confianza y de transparencia, sino también una correspondencia entre los elementos básicos de la formación en los distintos sistemas de educación superior.

Un trabajo serio y articulado en las cuatro líneas propuestas redundará en el fomento de la transparencia de los perfiles profesionales y académicos, en el de las titulaciones y en el de los programas de estudio, y favorecerá un énfasis cada vez mayor en los resultados. Así, la idea de que los estudiantes adquieran más competencias concretas afectará positivamente la transparencia en la definición de los objetivos fijados para un programa educativo específico. Esto se logrará añadiendo indicadores que puedan ser medidos con detalle, mientras se establece que esos objetivos tienen que ser dinámicos y acordes con las necesidades de la sociedad y del empleo. Tales cambios Ilevarán casi siempre a 
una transformación en el enfoque de las actividades educativas, puesto que propiciarán la participación sistemática del estudiante, ya sea como sujeto individual o como grupo, en la preparación de trabajos pertinentes, en presentaciones, etcétera.

El interés en el desarrollo de competencias en los programas educativos concuerda con un enfoque de la educación centrado ante todo en el alumno y en su capacidad de aprender, exigiendo más protagonismo y cuotas más altas de compromiso, ya que es el estudiante el que debe desarrollar las capacidades, las habilidades y las destrezas. Así mismo, se posibilitará la innovación a través de la elaboración de nuevos materiales de enseñanza, que ampararán tanto a los estudiantes como a los profesores, facilitando Ips procesos de enseñanza, aprendizaje y evaluación.

\section{4. ¿CÓMO SE IMPLEMENTARÁ EL PROYECTO?}

Hasta aquí hemos desgranado el núcleo de la metodología Tuning trasladada al espacio latinoamericano, pero la pregunta que surge ahora es: ¿como se llevará adelante el proyecto? En primer lugar, hay que resaltar que dicho proyecto es de las universidades para las universidades. Ellas seguirán siendo los actores principales en el debate y las que marcarán el ritmo del proceso. Habrá un total de 62 universidades latinoamericanas debatiendo en cuatro grupos de trabajo. Inicialmente se han definido cuatro áreas, a las que se incorporarán otras áreas de sinergia en el desarrollo del proyecto. Esas áreas serán: Administración de Empresas, Educación, Historia y Matemáticas. Las 62 universidades, pertenecientes a los 18 países de América Latina (Argentina, Bolivia, B rasil, Chile, Colombia, Costa Rica, Cuba, Ecuador, El Salvador, Guatemala, Honduras, México, Nicaragua, Panamá, Paraguay, Perú, U ruguay y Venezuela) estarán repartidas en los cuatro grupos de trabajo.

Las universidades participantes serán seleccionadas por los ministerios de educación y/o por las conferencias de rectores de cada uno de los países, en función de los siguientes criterios: excelencia nacional en el área que representen, capacidad de diálogo con las personas de otras instituciones que trabajen la misma área, tener un peso significativo en el sistema (tamaño de la institución, trayectoria, credibilidad y autoridad académica), de tal manera que una parte importante del sistema quede representado en la participación de la institución. 
El funcionamiento del proyecto está garantizado por el Programa ALFA, y tanto la organización y la realización de las reuniones de trabajo, los desplazamientos de académicos, la manutención y la elaboración de documentos de discusión como la publicación de resultados y de informes, están previstos con financiamiento directo de la Comisión Europea. Las universidades implicadas participantes en el proyecto aportarán, en concepto de cofinanciamiento, el tiempo que le dediquen a su participación en las reuniones previstas los académicos que las representen.

\section{5. ¿QUÉ DIFERENCIAS HAY ENTRE TUNING-EUROPA Y TUNING-AMÉRICA LATINA?}

Ya hemos planteado los aspectos prácticos y de funcionamiento del proyecto, y la pregunta que resta es: ¿que aspectos son diferentes entre el proyecto que se desarrolla en Europa y esta propuesta de América Latina? Quizás la más importante se encuentre en el momento político que vive Europa y aquel por el que transita América Latina. Europa ha definido, después de años de lucha y de debate, un proyecto común. La Unión Europea, cada vez más amplia, diversa y colorista, avanza segura en un proceso de integración pleno, en el que la educación superior, como espacio común, tiene una fecha de concreción. Los ministros de educación se han comprometido a alcanzar el sueño de la Europa unida en materia de educación superior en el año 2010. América Latina, en estos momentos, avanza en diferentes procesos de integración regional 0 subregional a ritmos diversos.

Europa ha necesitado varias décadas para poder consolidar su proceso de integración. América Latina ha avanzado mucho en los últimos años, pero precisa afianzar aún más sus logros, aunque hay avances y existen experiencias ${ }^{5}$ que tienden a la integración en materia de educación superior. La inquietud está presente en América Latina, tal vez no tan explícita como la que se lleva adelante en Europa, pero con la dificultad añadida de no contar con el marco supranacional que brinda la Unión Europea.

${ }^{5}$ El Convenio Andrés B ello ( $C A B$ ), del que forman parte Bolivia, Chile, Colombia, Ecuador, España, Panamá, Perú y Venezuela, busca entre sus objetivos la integración educativa. EI MERCOSUR, junto con Bolivia y Chile, están trabajando en la implementación de un Mecanismo Experimental de Acreditación de carreras de grado (MEXA). 
La diferencia entre ambos continentes hace que la forma de ejecutar el proyecto tenga también disparidades entre uno y otro. EI marco común, los lineamientos establecidos por B olonia, los compromisos contraídos, hacen que el proyecto en Europa tenga un marco más preciso. En el proyecto europeo participan actualmente más de 135 universidades, pero el propio ritmo del proceso hace que las instituciones de educación superior se entreguen a discutir, a pensar y a repensar los resultados alcanzados por el proyecto. Las universidades de Europa son parte del proceso que las Ilevará, a partir del 2010, a compartir el mismo espacio de educación superior. Y este compromiso externo redundará en una toma de conciencia y en un fuerte compromiso interno con el proceso, tanto a nivel nacional como supranacional. Las instituciones de educación superior europeas viven el desafío de Bolonia para el 2010, y de ahí que se involucren a un nivel tan profundo con los cambios que hay que llevar adelante para la concreción de este desafío.

América Latina no tiene fecha para determinar un espacio de educación superior; tampoco cuenta con un marco político tan encaminado como el que posee Europa. El proyecto Tuning-América Latina no pretende crear ese espacio, pero sí quisiera favorecer su creación. La potestad del debate seguirá siendo de las universidades, pero el proyecto ha buscado darle un espacio en paralelo al de los responsables de la política universitaria, para encontrarse y para aprovechar los momentos de discusión que las instituciones de educación superior de su país comparten con sus homólogas de otros países. Esos momentos de reflexión conjunta pueden despejar obstáculos, encontrar puntos de contacto, identificar aspectos positivos en el otro, resolver, acordar, pero, sobre todas las cosas, dialogar. Entender al otro, y con el otro tratar de construir algo en común. El proyecto pretende reunir a los responsables de la política universitaria de los 18 países latinoamericanos para dialogar, para conversar, para discutir sobre las potencialidades de pensar más allá de las fronteras nacionales, observando cómo las acuerdos alcanzados por los académicos de los grupos de trabajo del proyecto pueden favorecer los acercamientos entre los pueblos de la región, y estos, a su vez, posicionar mejor el sistema regional frente a otros.

De esa forma, en el proyecto Tuning-América Latina, además de las universidades, habrá un segundo actor rel evante que será el formado por los Centros Nacionales Tuning (CNT). Cada país de América Latina participará en el proyecto en igualdad de condiciones, a través de estos centros nacionales. El espíritu Tuning consiste en que está abierto a 


\section{GRÁFICO}

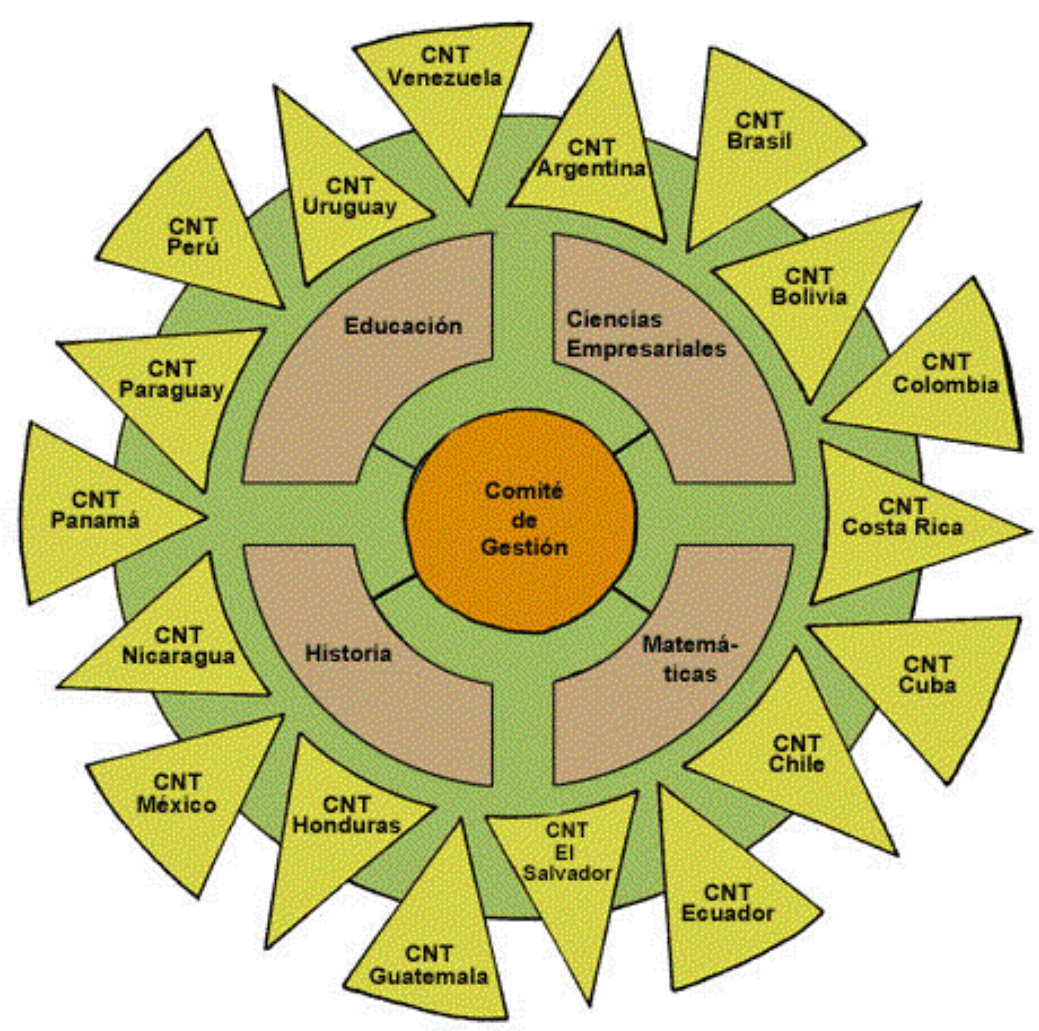

todas las voces, que se incluye a todos los países, por pequeños que sean. Los 18 tendrán una participación en el proyecto haciendo su aportación al desarrollo de la metodología en Latinoamérica. Tuning-América Latina estará liderado por las universidades, que son los actores clave en este proceso. Ios centros nacionales acompañarán a las instituciones en este camino, fortaleciendo en algunos casos su andar, y, sobre todas las cosas, le darán voz al sistema de educación superior en su conjunto. Su responsabilidad radica en ser interlocutor de su sistema dentro del proyecto; en sumar al diálogo los aportes de todos los actores que componen su sistema nacional; en escuchar y en transmitir estos mensajes; y en ser el interlocutor, el nexo entre el proyecto y el país. 


\section{CONCLUSIÓN}

Una vez culminados los dos años de intenso trabajo previstos en Tuning-América Latina, esperamos encontrarnos frente a un sistema educativo más sensible a los nuevos debates, en el que se hayan discutido en profundidad, y a nivel de las áreas del conocimiento, aspectos centrales como la definición de perfiles a través de competencias. Las instituciones participantes que han discutido y consensuado resultados del proyecto, ya no serán las mismas.

Basados en la experiencia, creemos que este debate es el inicio de un proceso de cambio y de mejora para las instituciones como ejes del sistema, pero también para el sistema en su conjunto. Los grados de avance a escala nacional dependerán del compromiso que asuman los responsables políticos de los centros nacionales a la hora de abordar nuevas propuestas y de implementar mejoras para la totalidad del sistema. Las propias instituciones participantes, con la experiencia alcanzada en este debate, deberán trabajar en mancomunidad a nivel nacional y regional para trasladar la discusión a las demás instituciones. Creemos que, finalizado el proceso, las estructuras educativas no sólo serán más transparentes sino también más dinámicas, favoreciendo la movilidad y el encuentro dentro de América Latina, tendiendo a su vez los puentes necesarios con Europa y con otras regiones del planeta. Nos gustaría pensar que, como en el caso de Europa, este es un proceso de cambio y de mejora.

«Tuning es una iniciativa de las universidades. Propuesta por las universidades, pero es una iniciativa en la que nosotros creemos [...] ¿Por qué el proyecto Tuning está recibiendo tanta atención y creando tantas expectativas? Yo creo que es porque el proyecto Tuning está en el corazón del proceso de Bolonia y se dirige a varias de sus líneas de acción: titulaciones comprensibles y comparables, adopción de un sistema de créditos, aseguramientocelacalidads.

Creemos que el aporte y la riqueza de los académicos y de las instituciones latinoamericanas contribuirán mucho a la creación de puentes y de lenguajes comunes que nos hagan valorar y reconocer

\footnotetext{
${ }^{6}$ Viviane Reding, comisaria de Educación y Cultura de la Comisión Europea. Discurso pronunciado en la Conferencia de Cierre del Proyecto Tuning Fase 1, Bruselas, 31/05/2002 (http://www.relint.deusto.es/TUNINGProject/index.htm).
} 
aquello que merece la pena, que puede mejorarnos a todos, y que existe en cualquier lugar de nuestro mundo.

\section{BIBLIOGRAFÍA}

BERGAN, S. (2003): «Qualification Structures in Higher Education», Danish Bologna Seminar, Copenhague, 27-28 de marzo.

COMMISSION OF THE EUROPEAN COMMUNITIES (2003): «The R ole of the Universities in the Europe of Knowledge», Communication from the Commission, COM (2003) 58 Final, Bruselas, 5 de febrero.

GONZÁLEZ, J . y WAGENAAR, R. (2003): Tuning Educational Structures in Europe. Informe Final - Proyecto Piloto, Fase 1, Bilbao, Universidad de Deusto.

DECLARACIÓN DE BOLONIA y otros documentos relevantes del proceso, en http:// www. bologna-berlin2003.de/en/main_documents/index. htm. 


\title{
Contactar
}

Revista lberoamericana de Educación

\author{
Principal OEI
}

